\title{
Mechanism of M-cell differentiation accelerated by proliferation of indigenous bacteria in rat Peyer's patches
}

\author{
Hideto YUASA ${ }^{1)}$, Youhei MANTANI ${ }^{1}$, Natsumi MASUDA ${ }^{1)}$, Miho NISHIDA ${ }^{1)}$, \\ Masaya ARAI ${ }^{1)}$, Toshifumi YOKOYAMA ${ }^{2)}$, Hiroki TSURUTA ${ }^{3)}$, Junichi KAWANO4), \\ Nobuhiko $\mathrm{HOSH}^{2)}$ and Hiroshi KITAGAWA ${ }^{1) *}$ \\ 1)Laboratory of Histophysiology, Department of Bioresource Science, Graduate School of Agricultural Science, \\ Kobe University, 1-1 Rokkodai-cho, Nada-ku, Kobe, Hyogo 657-8501, Japan \\ 2)Laboratory of Molecular Morphology, Department of Bioresource Science, Graduate School of Agricultural \\ Science, Kobe University, 1-1 Rokkodai-cho, Nada-ku, Kobe, Hyogo 657-8501, Japan \\ ${ }^{3)}$ Center for Collaborative Research and Technology Development, Kobe University, 1-1 Rokkodai-cho, Nada-ku, \\ Kobe, Hyogo 657-8501, Japan \\ 4)Laboratory of Microbiology and Immunology, Department of Bioresource Science, Graduate School of \\ Agricultural Science, Kobe University, 1-1 Rokkodai-cho, Nada-ku, Kobe, Hyogo 657-8501, Japan
}

J. Vet. Med. Sci.

79(11): 1826-1835, 2017

doi: 10.1292/jvms.17-0470

Received: 21 August 2017

Accepted: 24 September 2017

Published online in J-STAGE:

8 October 2017
ABSTRACT. The mechanism by which indigenous bacteria on the follicle-associated epithelium (FAE) of lymphatic follicles (LFs) accelerate the differentiation of microvillous columnar epithelial cells (MV) into M-cells was immunohistochemically investigated in rat Peyer's patches. The results showed that the number of Toll-like receptor (TLR) $-4^{+} M$-cells was greater in the FAE with expansion of bacterial colonies (LFs with bacterial colonies on the FAE: b-LF) than the FAE without expansion of bacterial colonies (nb-LF). TLR- 4 was also expressed in the striated borders of MV upstream next to M-cells in the FAE of the b-LF. TLR-4 ${ }^{+}$vesicles were frequently detected in the cytoplasms of MV with TLR-4 $4^{+}$striated borders upstream next to TLR-4+ $\mathrm{M}$-cells in the FAE of b-LF. These findings suggest that TLR-4+ MV take up TLR-4 ligands and differentiate into M-cells in the b-LF. Neither the distribution of RANK nor that of RANKL was coincident with that of M-cells in the b-LF. Moreover, RANK, but not RANKL, was expressed in intestinal villi, whereas cleaved caspase-3 was immunonegative in the MV and M-cells of the FAE, unlike in villous epithelial cells. Therefore, RANK/RANKL signaling in the LF might contribute to the down-regulation of epithelial apoptosis to facilitate the differentiation of MV into M-cells in rat Peyer's patches.

KEY WORDS: immunohistochemistry, indigenous bacteria, M-cell differentiation, Peyer's patch, rat

Numerous indigenous bacteria reside in the alimentary tract of animals. The settlement of these bacteria is known to be regulated by IgA, based on the finding that a deficiency of activation-induced cytidine deaminase, which induces IgA, causes a change in bacterial number and composition in the mouse small intestine [7, 23]. The immune response of IgA is induced in gutassociated lymphatic tissue (GALT) such as the Peyer's patches, which are aggregates of lymphatic tissue in the small intestine that contain several lymphatic follicles (LFs) [21, 22]. It has been suggested that LFs probably monitor the development of bacterial colonies in the restricted areas around the LFs in the rat small intestine [27]. However, the effects of indigenous bacteria on the structure and function of LFs remain unknown.

An LF consists of a dome area, follicular area, parafollicular area and follicle-associated epithelium (FAE); the FAE is composed of predominant microvillous columnar epithelial cells (MV), rare goblet cells and a few M-cells [3, 4, 18, 28]. The M-cell is a specialized epithelial cell that functions in the transportation of antigenic substances from the intestinal lumen to the subepithelial immunocompetent cells, thereby initiating mucosal immune responses $[17,18]$. The uptake of indigenous bacteria by M-cells has been thought to contribute to the regulation of indigenous bacteria in the rat alimentary tract [4, 27]. Moreover, cellular differentiation of the MV of FAE into M-cells is stimulated by the colonization of indigenous bacteria on FAE in the rat Peyer's patches [16]. However, the mechanisms by which indigenous bacteria accelerate the M-cell differentiation remain unclear.

The cellular differentiation into M-cells is initiated by active pinocytosis of MV in the FAE of rat Peyer's patches [16]. In a previous study, based on the finding that stimulation from indigenous bacteria on the FAE activate the differentiation into 
M-cells [4], we speculated that the pinocytosis of MV for the differentiation into M-cells was related to the receptors for bacterial recognition, specifically the Toll-like receptors (TLR) -2, -4 and -9 [28], which recognize the peptidoglycan, lipoprotein and lipoteichoic acid (TLR-2), the lipopolysaccharide (TLR-4) and the unmethylated CpG sequences (TLR-9) in DNA, respectively $[1,12]$. However, no MV, which are the precursor cells of M-cells, express TLR-2, -4 or -9 in the LFs that lack bacterial colonies on the FAE [28]. On the other hand, the expressions of TLRs in the epithelia are increased by stimulation with TLR-ligands in the murine colon $[6,14]$ and bovine mammary [11]. However, the expressions of TLRs in the LF with the hyperproliferation of indigenous bacteria on the FAE have not yet been investigated. Therefore, the first aim of this study was to immunohistochemically clarify whether TLR-2, -4 or -9 is involved with the acceleration of M-cell differentiation in the LFs exhibiting expansion of indigenous bacterial colonies on the FAE in rat Peyer's patches.

Recently, RANK/RANKL signaling has been suggested to be an important factor for the differentiation of MV into M-cells in RANK-knockout mice [19] or RANKL-knockout mice [13]. The induction of differentiation into M-cells by RANKL in vitro has been studied in the cultured cells derived from murine [5, 26], bovine [24] and human intestinal epithelial cells [20]. On the other hand, the down-regulation of epithelial apoptosis in the FAE is probably involved in the differentiation into M-cells [15]. Moreover, RANK/RANKL signaling is involved in the inhibition of epithelial apoptosis in the murine mammary gland [8, 10]. Therefore, a second goal of this study was to immunohistochemically clarify the relationship among RANK/RANKL signaling, down-regulation of epithelial apoptosis and the differentiation of MV into M-cells using LFs with or without expansion of indigenous bacterial colonies on the FAE in rat Peyer's patches.

\section{MATERIALS AND METHODS}

\section{Animals}

Twenty male SPF Wistar rats aged 7 weeks that were not littermates (Japan SLC, Hamamatsu, Japan) were maintained in an individually ventilated cage system (Tecniplast Japan, Tokyo, Japan) installed at the Kobe University Life Science Laboratory. Animals were permitted free access to water and food (Lab R-A2; Japan SLC). The animal facility was maintained under conditions of a $12 \mathrm{hr}$ light/dark cycle at $23 \pm 1^{\circ} \mathrm{C}$ and $50-60 \%$ humidity. Clinical and pathological examinations in all animals confirmed that there were no signs of disorder. This experiment was approved by the Institutional Animal Care and Use Committee (permission number: 25-06-01) and carried out according to the Kobe University Animal Experimentation Regulations.

\section{Tissue preparation}

After euthanasia with an overdose peritoneal injection of pentobarbital sodium (Kyoritsu Seiyaku, Tokyo, Japan), small tissue blocks with Peyer's patches were removed from the ileum. For the detection of TLR-2, -4 or -9 , the tissue blocks from 10 rats were immersion-fixed in $4.0 \%$ paraformaldehyde fixative in phosphate buffer ( $\mathrm{PB}$; pH 7.4) for $24 \mathrm{hr}$ at $4^{\circ} \mathrm{C}$. For the detection of RANK, RANKL or cleaved caspase-3, the tissue blocks from another 10 rats were immersion-fixed in $4.0 \%$ paraformaldehyde fixative in PB for $1 \mathrm{hr}$ at $4^{\circ} \mathrm{C}$. Then, all tissue blocks were snap-frozen in liquid nitrogen with reference to the embedding method described previously [28]. Four micrometer-thick sections were cut using a Coldtome CM1950 (Leica Biosystems, Nussloch, Germany) and were placed on slide glasses precoated with 2\% 3-aminopropyltriethoxysilane (Shin-Etsu Chemical, Tokyo, Japan) and stored at $-30^{\circ} \mathrm{C}$ until use.

\section{Immunohistochemistry}

Detection of antigens was conducted using the indirect method of enzyme immunohistochemistry. After three rinses with $0.05 \%$ Tween-added $0.01 \mathrm{M}$ phosphate buffered saline (TPBS; $\mathrm{pH}$ 7.4), the sections were immersed in absolute methanol with $0.5 \% \mathrm{H}_{2} \mathrm{O}_{2}$ for $30 \mathrm{~min}$. The sections were rinsed three times in TPBS after each preparation step to remove any reagent residues. Following blocking with Blocking One Histo (Nacalai Tesque, Kyoto, Japan) for $1 \mathrm{hr}$ at room temperature (r.t.), the sections were reacted with anti-TLR-2 (D-17), TLR-4 (M-16), TLR-9 (N-15) (diluted at 1:100; Santa Cruz Biotechnology, Santa Cruz, CA, U.S.A.) or RANKL (C-20) (diluted at 1:400; Santa Cruz Biotechnology) goat IgG, anti-RANK (B-8) mouse IgG (diluted at 1:100; Santa Cruz Biotechnology) or anti-cleaved caspase-3 rabbit IgG (diluted at 1:400; Cell Signaling Technology, Danvers, MA, U.S.A.) for 18 hr at $6^{\circ} \mathrm{C}$. The antibody specificities for rat TLR-2, -4, -9, RANK, RANKL and cleaved caspase-3 are described in the manufacturer's specification form (TLR-2, sc-12504; TLR-4, sc-12511; TLR-9, sc-13215; RANK, sc-390655; RANKL, sc-7627; cleaved caspase-3, \#9664S). Then, the sections were incubated with horseradish peroxidase-conjugated anti-goat IgG donkey IgG (diluted at 1:400; Jackson ImmunoResearch Laboratory, West Grove, PA, U.S.A.), anti-mouse IgG rat IgG (diluted at 1:100; Jackson ImmunoResearch Laboratory), and anti-rabbit IgG goat F (ab') ${ }_{2}$ (diluted at 1:200; Millipore, Billerica, MA, U.S.A.) for 1 hr at r.t. Finally, the sections were reacted with 3, 3'-diaminobenzidine (Dojindo Laboratories, Mashiki, Japan) containing $0.03 \% \mathrm{H}_{2} \mathrm{O}_{2}$ and were counterstained with hematoxylin. Control sections were incubated with TPBS or non-immunized goat IgG (Peprotech, Rocky Hill, NJ, U.S.A.), mouse IgG (Santa Cruz Biotechnology) and rabbit IgG (Alpha Diagnostic International, San Antonio, TX, U.S.A.) instead of the primary antibody.

\section{Histological detection of bacteria}

Briefly, the sections were stained with Gram's stain reagent solution 1 (Muto Pure Chemicals, Tokyo, Japan) for 2 min at r.t. After rinsing with distilled water, the sections were incubated with Gram's stain reagent solution 2 (Muto Pure Chemicals) for 1 min at r.t., followed by rinsing with absolute ethanol for $50 \mathrm{sec}$ and dehydration with acetone for $1 \mathrm{~min}$ at r.t. Then, the sections 


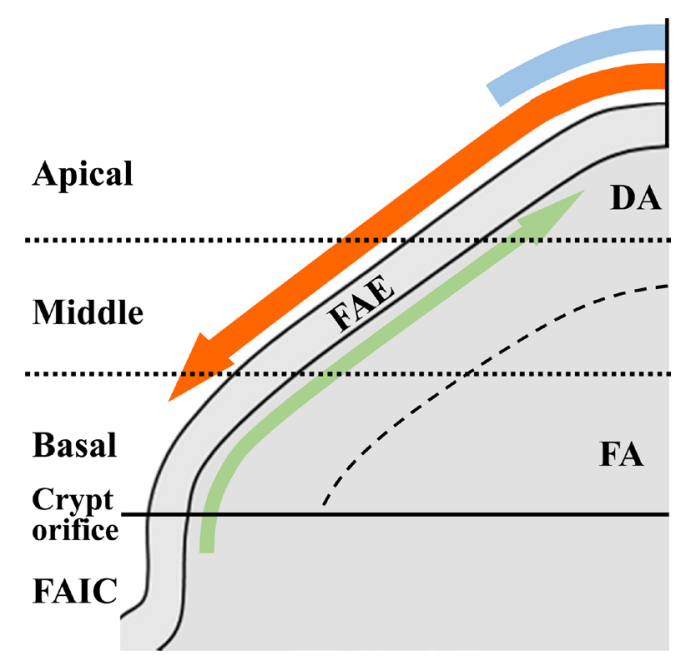

Fig. 1. Diagram of the left half of a rat lymphatic follicle (LF). The LF is divided into 3 portions, so that the follicle-associated epithelium (FAE) is also divided into basal, middle and apical portions. A green arrow indicates the stream of epithelial cell migration. A blue bar represents a bacterial colony restricted at the apical portion of the nb-LF and a red arrow represents the expansion of the bacterial colony from the apical portion in the b-LF. The dotted lines represent borders between each portion of the LF. The dashed line represents a border between the dome area (DA) and follicular area (FA). FAIC, follicle-associated intestinal crypt.

were incubated with Gram's stain reagent solution 3 (Muto Pure Chemicals) for 1 min at r.t. After rinsing with distilled water, the sections were dehydrated with acetone for $1 \mathrm{~min}$ at r.t.

\section{Definition of tissue elements and degree of proliferation of indigenous bacteria}

Sections from each tissue block were stained with hematoxylin-eosin for the observation of the general structure and the degree of proliferation of indigenous bacteria. The intestinal villus and LF, and thereby also the FAE, were divided into three portions from crypt orifice to apex of intestinal villus and LF: the basal, middle and apical portions. LFs were classified into two groups by degree of expansion of bacterial colonies on the FAE: nb-LF, which were LFs with no bacterial colonies on the FAE or with bacterial colonies only on the apical portions of the FAE, and b-LF, which were LFs with bacterial colonies from the apical to the middle or basal portions of the FAE (Fig. 1).

\section{Histoplanimetry}

Twenty nb-LFs and b-LFs whose central axes were longitudinally cut were randomly chosen from 10 rats. The numbers of M-cells were counted in the basal, middle or apical portions of FAE in the nb-LFs or b-LFs. The numbers of TLR-2 $2^{+},-4^{+}$or $-9^{+}$ MV and M-cells were also counted in each portion of the FAE in nb-LFs or b-LFs.

\section{Statistical analysis}

For the comparison of the number of M-cells, TLR-2 $2^{+},-4^{+}$or $-9^{+} \mathrm{MV}$ or TLR- $-2^{+},-4^{+}$or $-9^{+} \mathrm{M}$-cells between the FAE of nb-LF and b-LF, the normality of distribution was first assessed by the Kolmogorov-Smirnov test. For parametric variables, Welch's $t$-test was performed, and for non-parametric variables, a Mann-Whitney $U$-test was performed. $P$ values less than 0.05 were considered statistically significant.

\section{RESULTS}

\section{General histology}

In the Peyer's patch, M-cells with typical pockets were contained in the FAE, but never in the epithelia of follicle-associated intestinal villi and ordinary intestinal villi (Fig. 2a, 2a-1, 2b and 2b-1). Many M-cells were located in the basal and middle portions of the FAE of the nb-LF compared with the apical portion (Fig. 2a). The number of M-cells was greater throughout the FAE of the b-LF, especially in the apical portion, than the FAE of the nb-LF (Fig. $2 b$ and $2 c$ ).

\section{Characteristics of bacteria on the FAE of the LF}

Bacterial colonies were frequently observed on the FAE of LFs. Almost all of the bacteria on the FAE were Gram-negative bacilli (Fig. 3a). The bacteria on the FAE frequently adhered to MV in the apical portion of the LF (Fig. 3a-1).

\section{Distribution of TLRs in the $n b-L F$}

Almost all of the MV and goblet cells were immunonegative for TLR-2, -4 and -9 throughout the FAE of the nb-LF (Figs. 4a, 6a and 7a). The roofs of some M-cells were immunopositive for TLR-2, -4 and -9 throughout the FAE in the nb-LF (arrows in Figs. 4a-1, 6a-1 and 7a-1), but MV upstream next to M-cells were immunonegative for TLR-2, -4 and -9 in the FAE of nb-LF (arrowheads in Figs. 4a-1, 6a-1 and 7a-1).

Distribution of TLRs in the $b-L F$

Almost all of the MV were immunonegative for TLR-2 in the basal and middle portions of the FAE of the b-LF (Fig. 4b). 

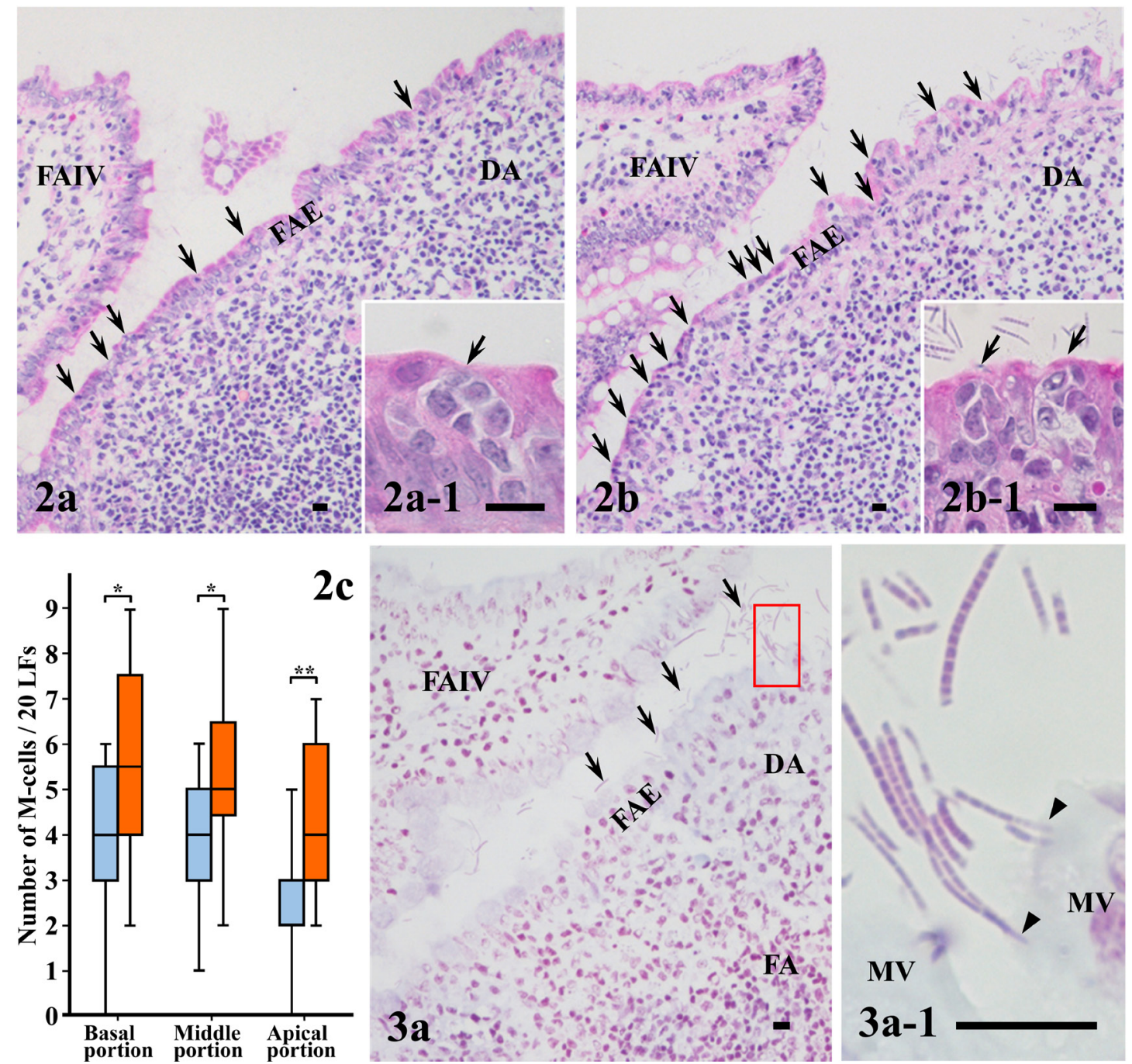

Fig. 2. Light microscopic distribution of M-cells in the follicle-associated epithelium (FAE) of the nb-LF (a) and b-LF (b). M-cells (arrows) have typical pockets with migrating cells (a-1 and b-1). c) The number of M-cells in the apical, middle and basal portions of the FAE in the nb-LF (blue column) and b-LF (red column). DA, dome area. FAIV, follicle-associated intestinal villus. The data are presented as Box and Whisker plots in which boxes extend from the first quartile to the third quartile with a black line at the median. Whiskers (slender bars) represent the highest or lowest values. Asterisks, $P<0.05$. Double asterisks, $P<0.01$. Bar $=10 \mu \mathrm{m}$.

Fig. 3. a) Gram's staining shows that all bacteria on the follicle-associated epithelium (FAE) are Gram-negative. a-1) High-magnification image of the red square in (a). Gram-negative bacteria adhere to the microvillous columnar epithelial cells (MV) in the apex of the FAE (arrowheads). $\mathrm{DA}$, dome area. FA, follicular area. FAIV, follicle-associated intestinal villus. Bar $=10 \mu \mathrm{m}$.

The roofs of some M-cells were immunopositive for TLR-2 throughout the FAE in the b-LF, but MV upstream next to M-cells were immunonegative for TLR-2 in the FAE of the b-LF (Fig. 4b-1). TLR-2-immunopositivities were frequently detected in the striated borders of MV in the apical portion of the FAE of the b-LF. Most of MV which indigenous bacteria adhered to were immunopositive for TLR-2 in the apical portion of the b-LF (arrowheads in Fig. 4b-2). TLR-2 $2^{+}$granules were rarely found in the cytoplasms of MV in the basal and middle portions of the FAE of the b-LF, and were detected in moderate numbers in the cytoplasms of MV in the apical portions in the b-LF (Fig. 4b-1 and 4b-2). The numbers of TLR-2 $2^{+}$MV were significantly greater in the apical portion of the FAE of the b-LF than in that of the nb-LF (Fig. 5a). The numbers of M-cells with TLR-2 $2^{+}$roofs were not significantly different in the apical, middle or basal portions between the nb-LF and b-LF (Fig. 5b).

TLR-4-immunopositivity was detected in the striated borders of MV throughout the FAE of the b-LF (Fig. 6b). The roofs of some M-cells were immunopositive for TLR-4 throughout the FAE in the b-LF, and MV upstream next to M-cells in the FAE were immunopositive for TLR-4 in the b-LF (Fig. 6b-1). Moreover, TLR-4 ${ }^{+}$vesicles of various sizes were frequently detected in the cytoplasms of TLR-4 $4^{+}$MV throughout the FAE in the b-LF (dashed circle in Fig. 6b-1). TLR-4-immunopositivity was detected in the striated borders of most of MV in the apical portion of the FAE in the b-LF (Fig. 6b-2). Most of MV which indigenous bacteria adhered to were immunopositive for TLR-4 in the apical portion of the b-LF (arrowheads in Fig. 6b-2). The numbers of 


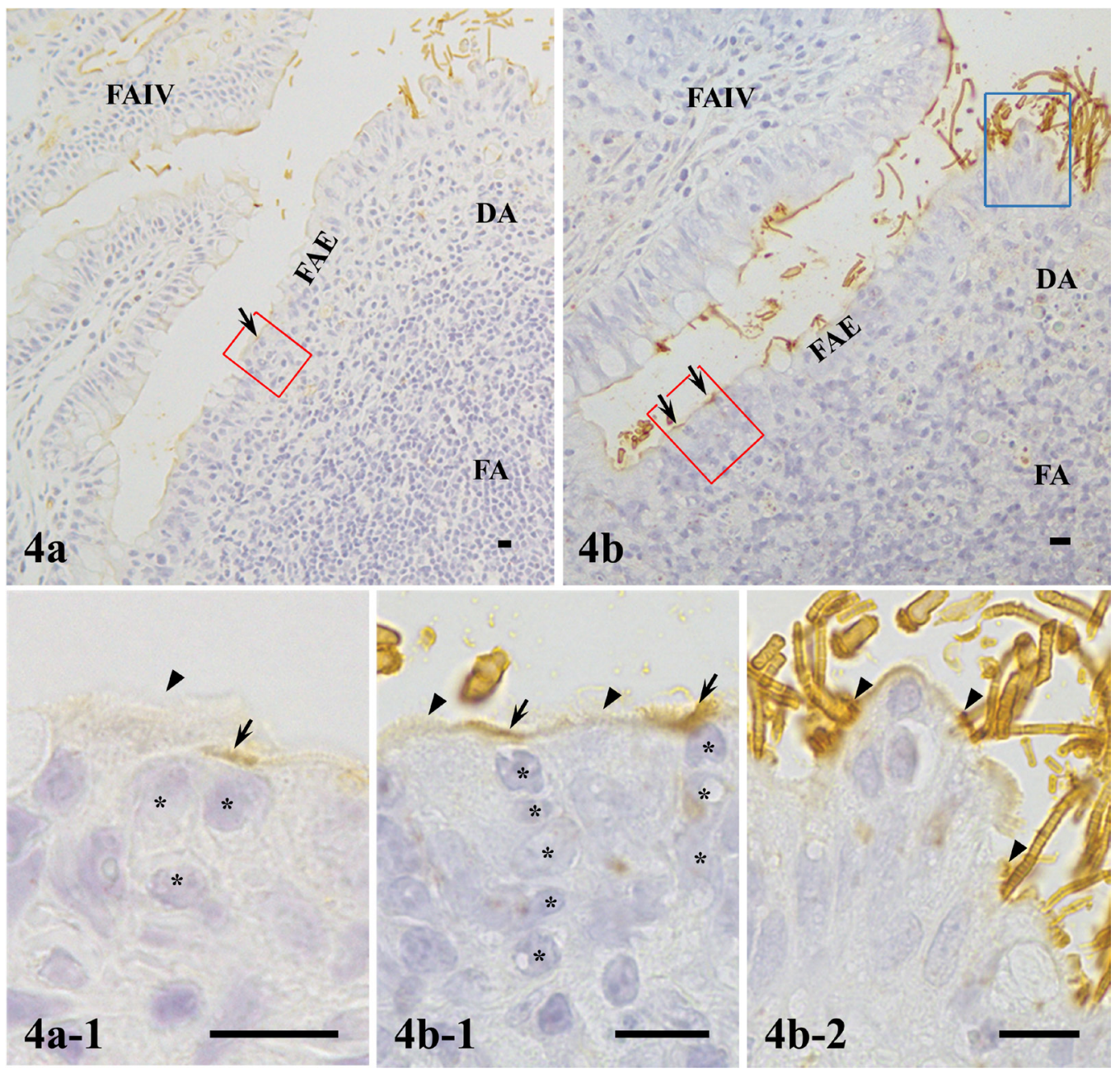

Fig. 4. Distribution of TLR-2 in the follicle-associated epithelium (FAE) of the nb-LF (a) and b-LF (b). a-1), b-1) and b-2) are high-magnification images of red boxes or a blue box in (a) and (b). The microvillous columnar epithelial cells (MV) (arrowheads) upstream next to TLR-2 ${ }^{+}$M-cell (arrows) are immunonegative for TLR-2 in the nb-LF (a-1) and b-LF (b-1). b-2) Indigenous bacteria (arrowheads) adhere to the TLR-2 ${ }^{+}$MV in the apical portion of the b-LF. Asterisks indicate the nuclei of migrating cells in typical pockets of TLR-2 ${ }^{+}$M-cells (arrows). DA, dome area. FA, follicular area. FAIV, follicle-associated intestinal villus. Bar $=10 \mu \mathrm{m}$.

TLR $-4^{+}$MV were significantly greater in the apical, middle and basal portions of the FAE in the b-LF than in those in the nb-LF, respectively (Fig. 5c). The number of M-cells with TLR- $4^{+}$roofs was significantly greater in the apical, middle and basal portions of the FAE in the b-LF than in those in the nb-LF, respectively (Fig. 5d).

Almost all of the MV were immunonegative for TLR-9 in the basal and middle portions of the FAE in the b-LF (Fig. 7b). The roofs of some M-cells were immunopositive for TLR-9 throughout the FAE in the b-LF, but MV upstream portion of M-cells in the FAE were immunonegative for TLR-9 in the b-LF (Fig. 7b-1). TLR-9-immunopositivities were moderately detected in the striated borders of MV in the apical portion of the FAE in the b-LF (Fig. 7b-2). Most of MV which indigenous bacteria adhered to were immunopositive for TLR-9 in the apical portion of the b-LF (arrowhead in Fig. 7b-2). TLR-9+ granules were rarely found in the cytoplasms of MV in the basal and middle portions of the FAE in the b-LF, and were detected in moderate numbers in the cytoplasms of MV in the apical portions of the FAE in the b-LF (Fig. 7b-1 and 7b-2). The number of TLR-9 $9^{+}$MV was significantly greater in the apical portion of the FAE in the b-LF than that in the nb-LF (Fig. 5e). The number of M-cells with TLR-9 $9^{+}$roofs in the apical, middle or basal portions was not significantly different between the nb-LF and b-LF (Fig. 5f).

\section{Distribution of RANK in the intestinal villus, $n b-L F$ and $b-L F$}

The epithelium of intestinal villi was immunopositive for RANK (Fig. 8a). The immunopositive intensity for RANK was moderate in the epithelium of the basal portion and gradually weakened toward the villous apex in the intestinal villus (Fig. 8a). The migrating cells in the lamina propria were frequently immunopositive for RANK in the intestinal villus (Fig. 8a-1). The MV and M-cells were moderately immunopositive for RANK throughout the FAE in the nb-LF and b-LF (Fig. 8b, 8b-1, 8c and 8c-1). Most of intraepithelial migrating cells were immunopositive for RANK in the nb-LF and b-LF (asterisks in Fig. 8b-1 and 8c-1). 

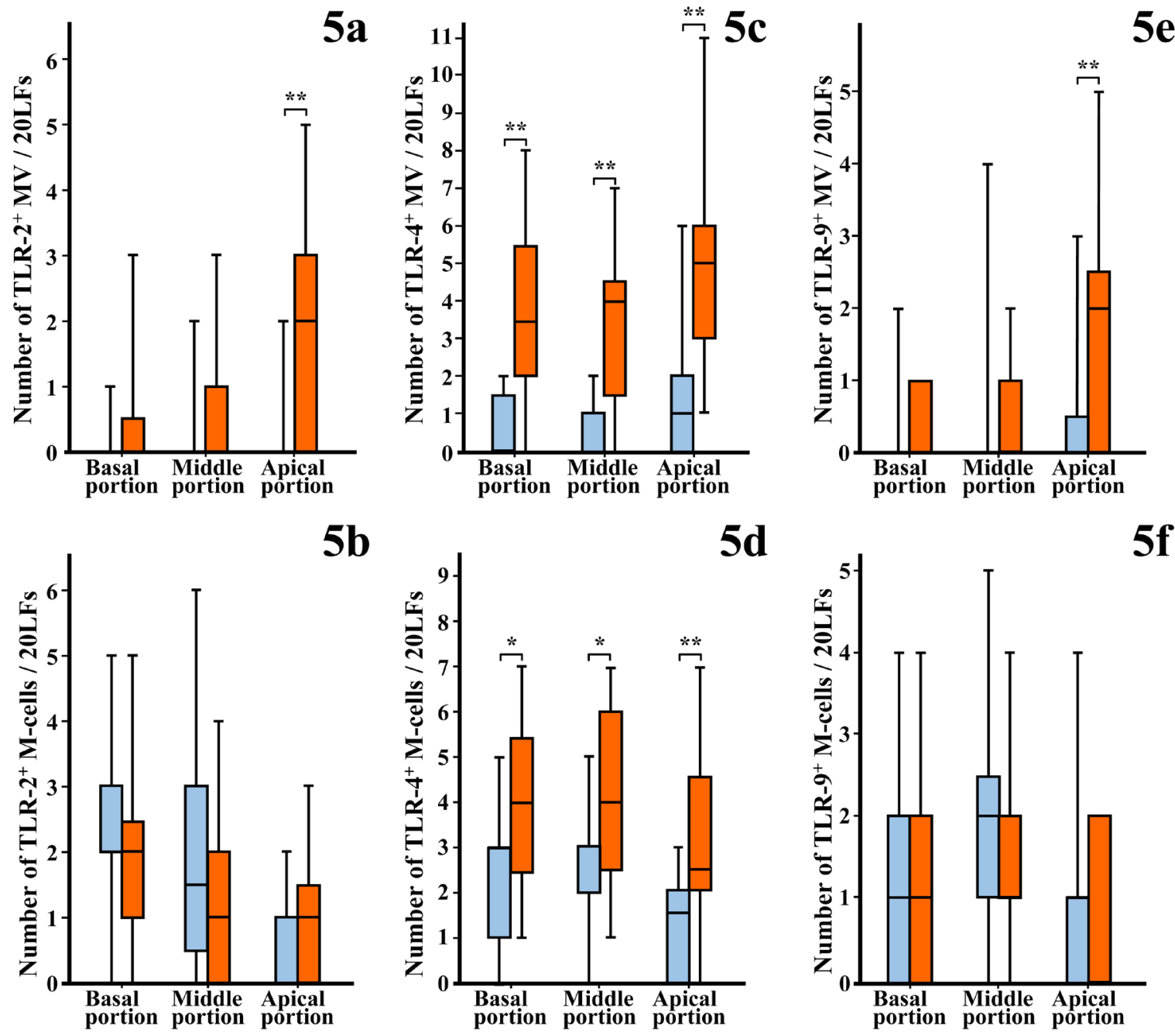

Fig. 5. The number of microvillous columnar epithelial cells (MV) (a, c and e) or M-cells (b, $d$ and $f$ ) immunopositive for TLR-2 (a and b), TLR-4 (c and d) and TLR-9 (e and f) in the follicle-associated epithelium of the apical, middle and basal portions of the nb-LF (blue columns) and b-LF (red columns). The data are presented as Box and Whisker plots in which boxes extend from the first quartile to the third quartile with a black line at the median. Whiskers (slender lines) indicate the highest or lowest values. Asterisks, $P<0.05$. Double asterisks, $P<0.01$.

\section{Distribution of RANKL in the intestinal villus, $n b-L F$ and $b-L F$}

The intestinal villi were immunonegative for RANKL (Fig. 9a). FAE were almost immunonegative for RANKL in the nb-LFs and b-LFs (Fig. 9b and 9c). The stromal cells in the subepithelial dome area were immunopositive for RANKL in the nb-LFs and b-LFs (dashed line in Fig. 9b and 9c). The immunopositive intensity for RANKL was strong beneath the FAE and gradually weakened toward the follicular area in the nb-LF and b-LF. The area of accumulated RANKL-immunopositive stromal cells was thick in the apical portion and became gradually narrow toward the basal portion in the nb-LF (Fig. 9b). The area with RANKL stromal cells in the basal portion of the b-LF was thicker than that in the basal portion of the nb-LF (Fig. 9b and 9c).

\section{Distribution of cleaved caspase-3 in the epithelia of the intestinal villus, $n b-L F$ and $b-L F$}

Immunopositivity for cleaved caspase-3 was detected in the cytoplasms of all MV and goblet cells throughout the intestinal villi and was especially strong in the MV of the apical portion of intestinal villi (Fig. 10a). The MV and M-cells were almost immunonegative for cleaved caspase-3 throughout the FAE in the nb-LF and b-LF except for a few MV in the apical portion (Fig. $10 \mathrm{~b}, 10 \mathrm{~b}-1,10 \mathrm{c}$ and $10 \mathrm{c}-1)$.

No immunopositivity was detected in any of the control sections.

\section{DISCUSSION}

The differentiation into M-cells is performed by the active pinocytosis of immature MV [16] and is accelerated by the increase of indigenous bacteria on the FAE in rat Peyer's patches [4]. In the present study, TLR-4 was frequently immunopositive in the 


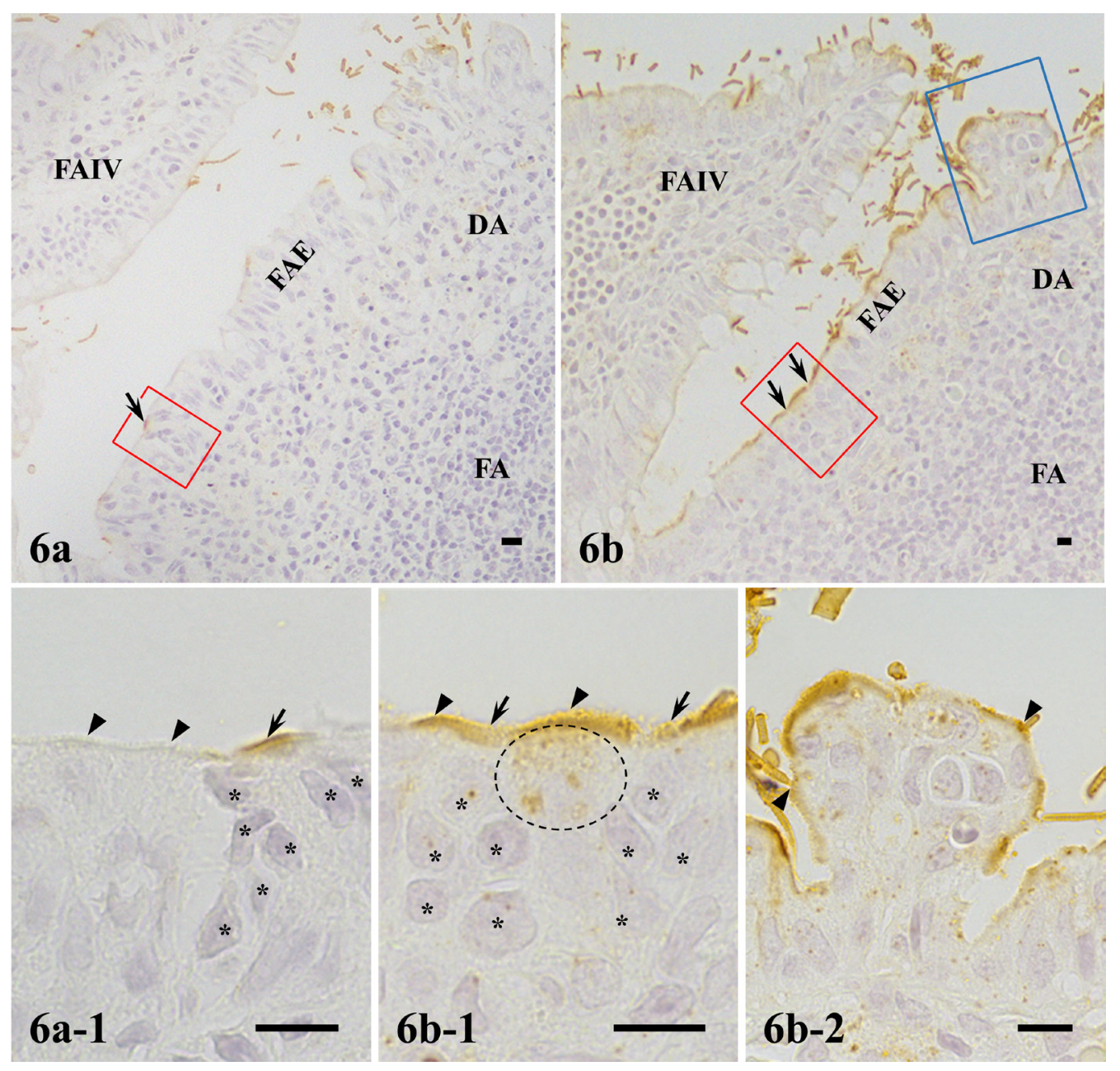

Fig. 6. Distribution of TLR-4 in the follicle-associated epithelium (FAE) of the nb-LF (a) and b-LF (b). a-1), b-1) and b-2) are high-magnification images of the red boxes or the blue box in (a) and (b). The microvillous columnar epithelial cells (MV) (arrowheads) upstream next to TLR-4 ${ }^{+}$ M-cell (arrow) are immunonegative for TLR-4 in the nb-LF (a-1), and immunopositive in the b-LF (b-1). TLR-4 ${ }^{+}$vesicles of various sizes are visible in the cytoplasms of TLR- $4^{+}$MV in the dashed circle (b-1). b-2) Indigenous bacteria (arrowheads) adhere to TLR-4 $4^{+}$MV in the apical portion of b-LF. Asterisks indicate the nuclei of migrating cells in typical pockets of TLR-4 $4^{+}$M-cells (arrows). DA, dome area. FA, follicular area. FAIV, follicle-associated intestinal villus. Bar $=10 \mu \mathrm{m}$.

striated borders of MV in the basal and middle portions of the FAE in the b-LF, whereas TLR-2 and -9 were immunonegative in MV, which are the precursor cells of M-cells, in the basal and middle portions of the b-LF. In addition, TLR- $4^{+}$vesicles were frequently detected in the cytoplasms of the MV with TLR $-4^{+}$striated borders in the basal and middle portions of the FAE in the b-LF. Considering that the number of TLR-4 $4^{+}$M-cells was greater in the FAE of b-LF than the FAE of nb-LF, these findings suggest that the colonization of indigenous bacteria on FAE induce an increase of TLR-4-expression, but not TLR-2 and -9, in MV, leading to the active uptake of TLR-4-ligand by MV and acceleration of M-cell differentiation in the FAE. Because the uptake of indigenous bacteria by M-cells has been thought to contribute to the regulation of indigenous bacteria in the rat alimentary tract [4, 27], the TLR-4-mediated increase of M-cells by indigenous bacteria might contribute to efficient uptake of indigenous bacteria by M-cell, leading to the regulation of indigenous bacteria in the alimentary tract.

It has been established that M-cells are dramatically decreased in the FAE of Peyer's patches in RANK- [19] and RANKLknockout mice [13]. RANKL induces the expression of an M-cell marker, GP2, in the cultured M-cells derived from murine [5, 26], bovine [24] and human epithelial cells [20]. In addition, RANK and RANKL are expressed in the FAE and the stromal cells in mouse Peyer's patches, respectively $[13,25]$. Therefore, it has been speculated that RANKL is an important factor for the induction of the differentiation into M-cells in the FAE $[13,19,20]$. In the present study, however, the intensity of immunopositivity for RANK was moderate throughout the FAE, and this pattern of intensity was the same in the b-LF. In addition, the area of accumulated RANKL-immunopositive stromal cells was thicker in the dome area of the apical portion than in the dome area of the basal portion in the nb-LF, whereas M-cells frequently appeared in the basal and middle portions of the FAE in the nb-LF. Moreover, the area with accumulated RANKL-immunopositive stromal cells was thicker in the basal and middle portion of the dome area in the b-LF than that in the nb-LF, whereas M-cells were markedly increased in the apical portion of the FAE 


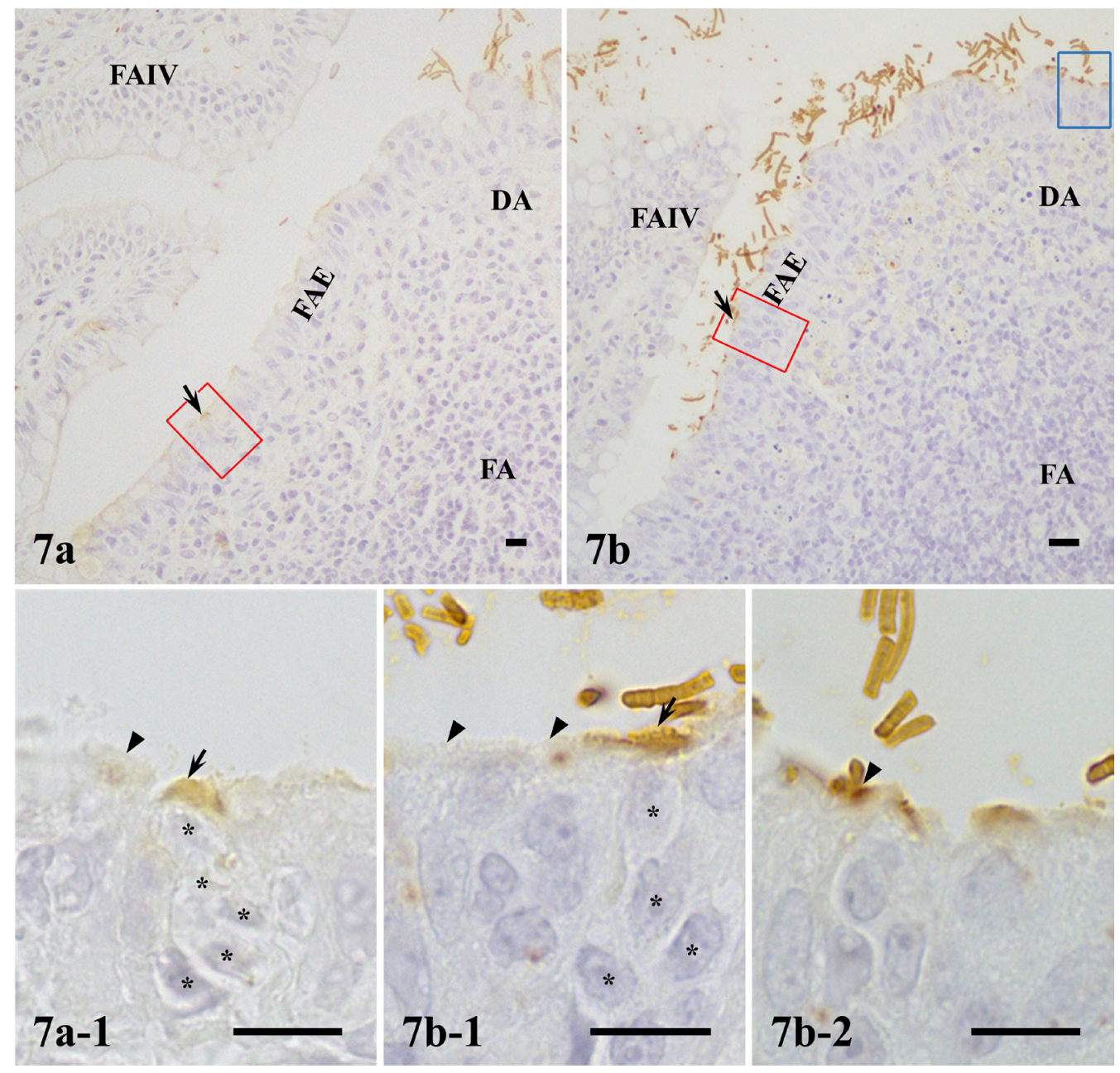

Fig. 7. Distribution of TLR-9 in the follicle-associated epithelium (FAE) of the nb-LF (a) and b-LF (b). a-1), b-1) and b-2) are high-magnification images of the red boxes or the blue box in (a) and (b). The microvillous columnar epithelial cells (MV) (arrowheads) upstream next to TLR-9 $9^{+}$ M-cell (arrows) are immunonegative for TLR-9 in the nb-LF (a-1) and b-LF (b-1). b-2) Indigenous bacterium (arrowhead) adhere to the TLR-9 ${ }^{+}$ MV in the apical portion of b-LF. Asterisks indicate the nuclei of migrating cells in typical pockets of TLR-9+ M-cells (arrows). DA, dome area. FA, follicular area. FAIV, follicle-associated intestinal villus. Bar $=10 \mu \mathrm{m}$.

of the b-LF compared to that in the nb-LF. These findings suggest that the distributions of RANK and RANKL were not fully correspondent with the distribution of M-cells in the nb-LF, and that neither distribution was correlated with the change in the distribution of M-cells in the b-LF. Therefore, RANK and RANKL probably do not participate directly in the differentiation into M-cells.

Caspase-3 plays a role not only in cellular apoptosis but also in the maturation of human erythroid progenitor cells in the early stages of erythroid differentiation [2,29] and the differentiation of murine myoblasts [9]. The expression of cleaved caspase-3 in the FAE of the rat LF occurs later than that in the epithelium of the intestinal villus [15]. These results suggest that the epithelial maturation of the FAE occurs later than the epithelial maturation in the intestinal villus, and this immaturity of FAE is probably required for the differentiation into M-cells in the rat small intestine [15]. In addition, previous reports have suggested that epithelial apoptosis is inhibited by RANK/RANKL signaling in the mammary gland $[8,10]$. In the present study, it was suggested that RANKL was expressed in the stromal cells of the LF, but not in those of the intestinal villus, and that almost none of the epithelial cells throughout the FAE, with the exception of those in the apex, expressed cleaved caspase-3 in the nb-LF and b-LF, whereas almost all of epithelial cells throughout the intestinal villus expressed cleaved caspase-3. Therefore, RANK/ RANKL signaling in the LF might contribute to the down-regulation of epithelial apoptosis to form a milieu suitable for M-cell differentiation.

ACKNOWLEDGMENT. This work was financially supported in part by Grants-in-Aid for Scientific Research (nos. $15 \mathrm{~K} 07766$ and 16K18813) from the Japan Society for the Promotion of Science. 


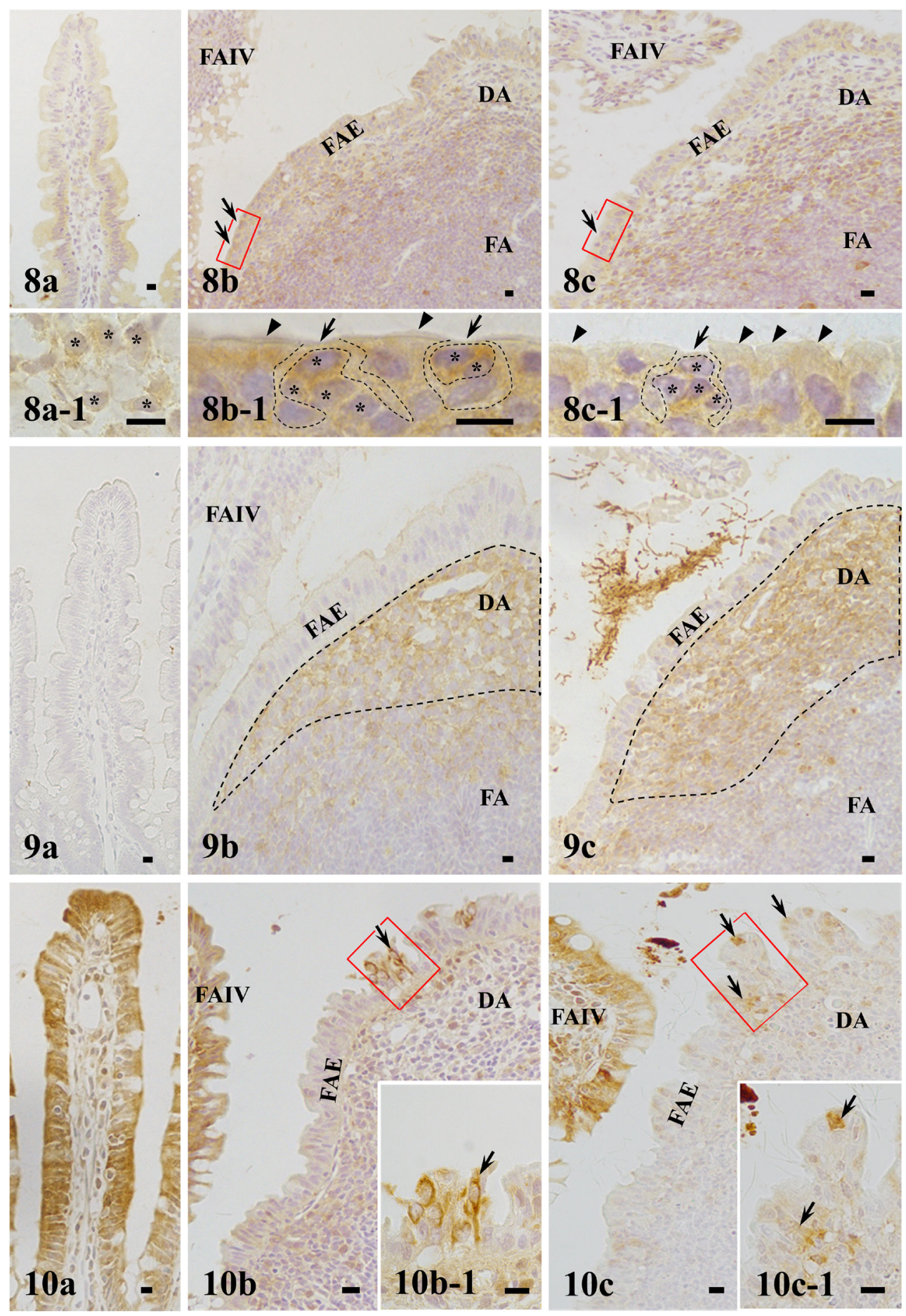

Fig. 8. Distribution of RANK in the intestinal villus (a), nb-LF (b) and b-LF (c). a, b) The intensity of immunopositivity for RANK in the epithelial cells is moderate in the basal portion of the intestinal villus and gradually weakens toward the villous apex. a-1) RANK ${ }^{+}$migrating cells (asterisks) exist in the lamina propria of the intestinal villus. b, c) M-cells (arrows) and other epithelial cells (arrowheads) are moderately immunopositive for RANK throughout the follicle-associated epithelium (FAE) of the nb-LF and b-LF. b-1) and c-1) are high-magnification images of the red boxes in (b) and (c). The dashed lines indicate the outlines of M-cells. Asterisks indicate the nuclei of migrating cells in typical pockets of RANK ${ }^{+}$M-cells (arrows) in (b-1) and (c-1). DA, dome area. FA, follicular area. FAIV, follicle-associated intestinal villus. Bar=10 $\mu \mathrm{m}$.

Fig. 9. Distribution of RANKL in the intestinal villus (a), nb-LF (b) and b-LF (c). a) The intestinal villus is immunonegative for RANKL. b, c) The accumulation of stromal cells is demarcated by dashed lines. DA, dome area. FA, follicular area. FAE, follicle-associated epithelium. FAIV, follicle-associated intestinal villus. Bar $=10 \mu \mathrm{m}$.

Fig. 10. Distribution of cleaved caspase-3 in the intestinal villus (a), nb-LF (b) and b-LF (c). a) All villous epithelial cells are immunopositive for cleaved caspase-3. b, c) The epithelial cells are cleaved caspase-3 immunonegative, except for microvillous columnar epithelial cells (MV) (arrows) in the follicle-associated epithelium (FAE) of the apical portions of the nb-LF and b-LF. b-1) and c-1) are high-magnification images of the red boxes in (b) and (c). Arrows indicate cleaved caspase-3 immunopositive MV. DA, dome area. FAIV, follicle-associated intestinal villus. Bar $=10 \mu \mathrm{m}$. 


\section{REFERENCES}

1. Akira, S., Uematsu, S. and Takeuchi, O. 2006. Pathogen recognition and innate immunity. Cell 124: 783-801. [Medline] [CrossRef]

2. Carlile, G. W., Smith, D. H. and Wiedmann, M. 2004. Caspase-3 has a nonapoptotic function in erythroid maturation. Blood 103: 4310-4316. [Medline] [CrossRef]

3. Cesta, M. F. 2006. Normal structure, function, and histology of mucosa-associated lymphoid tissue. Toxicol. Pathol. 34: 599-608. [Medline] [CrossRef]

4. Chin, K., Onishi, S., Yuji, M., Inamoto, T., Qi, W. M., Warita, K., Yokoyama, T., Hoshi, N. and Kitagawa, H. 2006. Differentiation of epithelial cells to M cells in response to bacterial colonization on the follicle-associated epithelium of Peyer's patch in rat small intestine. J. Vet. Med. Sci. 68: 1023-1028. [Medline] [CrossRef]

5. de Lau, W., Kujala, P., Schneeberger, K., Middendorp, S., Li, V. S. W., Barker, N., Martens, A., Hofhuis, F., DeKoter, R. P., Peters, P. J., Nieuwenhuis, E. and Clevers, H. 2012. Peyer's patch M cells derived from Lgr5 $\left(^{+}\right)$stem cells require SpiB and are induced by RankL in cultured "miniguts". Mol. Cell. Biol. 32: 3639-3647. [Medline] [CrossRef]

6. Ewaschuk, J. B., Backer, J. L., Churchill, T. A., Obermeier, F., Krause, D. O. and Madsen, K. L. 2007. Surface expression of Toll-like receptor 9 is upregulated on intestinal epithelial cells in response to pathogenic bacterial DNA. Infect. Immun. 75: 2572-2579. [Medline] [CrossRef]

7. Fagarasan, S., Muramatsu, M., Suzuki, K., Nagaoka, H., Hiai, H. and Honjo, T. 2002. Critical roles of activation-induced cytidine deaminase in the homeostasis of gut flora. Science 298: 1424-1427. [Medline] [CrossRef]

8. Fata, J. E., Kong, Y. Y., Li, J., Sasaki, T., Irie-Sasaki, J., Moorehead, R. A., Elliott, R., Scully, S., Voura, E. B., Lacey, D. L., Boyle, W. J., Khokha, R. and Penninger, J. M. 2000. The osteoclast differentiation factor osteoprotegerin-ligand is essential for mammary gland development. Cell 103: 41-50. [Medline] [CrossRef]

9. Fernando, P., Kelly, J. F., Balazsi, K., Slack, R. S. and Megeney, L. A. 2002. Caspase 3 activity is required for skeletal muscle differentiation. Proc. Natl. Acad. Sci. U.S.A. 99: 11025-11030. [Medline] [CrossRef]

10. Gonzalez-Suarez, E., Jacob, A. P., Jones, J., Miller, R., Roudier-Meyer, M. P., Erwert, R., Pinkas, J., Branstetter, D. and Dougall, W. C. 2010. RANK ligand mediates progestin-induced mammary epithelial proliferation and carcinogenesis. Nature 468: 103-107. [Medline] [CrossRef]

11. Ibeagha-Awemu, E. M., Lee, J. W., Ibeagha, A. E., Bannerman, D. D., Paape, M. J. and Zhao, X. 2008. Bacterial lipopolysaccharide induces increased expression of toll-like receptor (TLR) 4 and downstream TLR signaling molecules in bovine mammary epithelial cells. Vet. Res. 39: 11. [Medline] [CrossRef]

12. Kawai, T. and Akira, S. 2007. Signaling to NF-kappaB by Toll-like receptors. Trends Mol. Med. 13: 460-469. [Medline] [CrossRef]

13. Knoop, K. A., Kumar, N., Butler, B. R., Sakthivel, S. K., Taylor, R. T., Nochi, T., Akiba, H., Yagita, H., Kiyono, H. and Williams, I. R. 2009. RANKL is necessary and sufficient to initiate development of antigen-sampling M cells in the intestinal epithelium. J. Immunol. 183: 5738-5747. [Medline] [CrossRef]

14. Lundin, A., Bok, C. M., Aronsson, L., Björkholm, B., Gustafsson, J. A., Pott, S., Arulampalam, V., Hibberd, M., Rafter, J. and Pettersson, S. 2008. Gut flora, Toll-like receptors and nuclear receptors: a tripartite communication that tunes innate immunity in large intestine. Cell. Microbiol. 10: 1093-1103. [Medline] [CrossRef]

15. Onishi, S., Miyata, H., Inamoto, T., Qi, W. M., Yamamoto, K., Yokoyama, T., Warita, K., Hoshi, N. and Kitagawa, H. 2007a. Immunohistochemical study on the delayed progression of epithelial apoptosis in follicle-associated epithelium of rat Peyer's patch. J. Vet. Med. Sci. 69: $1123-1129$. [Medline] [CrossRef]

16. Onishi, S., Yokoyama, T., Chin, K., Yuji, M., Inamoto, T., Qi, W. M., Warita, K., Hoshi, N. and Kitagawa, H. 2007b. Ultrastructural study on the differentiation and the fate of M cells in follicle-associated epithelium of rat Peyer's patch. J. Vet. Med. Sci. 69: 501-508. [Medline] [CrossRef]

17. Owen, R. L. 1977. Sequential uptake of horseradish peroxidase by lymphoid follicle epithelium of Peyer's patches in the normal unobstructed mouse intestine: an ultrastructural study. Gastroenterology 72: 440-451. [Medline]

18. Owen, R. L. and Jones, A. L. 1974. Epithelial cell specialization within human Peyer's patches: an ultrastructural study of intestinal lymphoid follicles. Gastroenterology 66: 189-203. [Medline]

19. Rios, D., Wood, M. B., Li, J., Chassaing, B., Gewirtz, A. T. and Williams, I. R. 2016. Antigen sampling by intestinal M cells is the principal pathway initiating mucosal IgA production to commensal enteric bacteria. Mucosal Immunol. 9: 907-916. [Medline] [CrossRef]

20. Rouch, J. D., Scott, A., Lei, N. Y., Solorzano-Vargas, R. S., Wang, J., Hanson, E. M., Kobayashi, M., Lewis, M., Stelzner, M. G., Dunn, J. C. Y., Eckmann, L. and Martín, M. G. 2016. Development of functional microfold (M) cells from intestinal stem cells in primary human enteroids. PLOS ONE 11: e0148216. [Medline] [CrossRef]

21. Suzuki, K., Ha, S. A., Tsuji, M. and Fagarasan, S. 2007. Intestinal IgA synthesis: a primitive form of adaptive immunity that regulates microbial communities in the gut. Semin. Immunol. 19: 127-135. [Medline] [CrossRef]

22. Suzuki, K., Kawamoto, S., Maruya, M. and Fagarasan, S. 2010. GALT: organization and dynamics leading to IgA synthesis. Adv. Immunol. 107: 153-185. [Medline] [CrossRef]

23. Suzuki, K., Meek, B., Doi, Y., Muramatsu, M., Chiba, T., Honjo, T. and Fagarasan, S. 2004. Aberrant expansion of segmented filamentous bacteria in IgA-deficient gut. Proc. Natl. Acad. Sci. U.S.A. 101: 1981-1986. [Medline] [CrossRef]

24. Tahoun, A., Mahajan, S., Paxton, E., Malterer, G., Donaldson, D. S., Wang, D., Tan, A., Gillespie, T. L., O’Shea, M., Roe, A. J., Shaw, D. J., Gally, D. L., Lengeling, A., Mabbott, N. A., Haas, J. and Mahajan, A. 2012. Salmonella transforms follicle-associated epithelial cells into M cells to promote intestinal invasion. Cell Host Microbe 12: 645-656. [Medline] [CrossRef]

25. Taylor, R. T., Patel, S. R., Lin, E., Butler, B. R., Lake, J. G., Newberry, R. D. and Williams, I. R. 2007. Lymphotoxin-independent expression of TNF-related activation-induced cytokine by stromal cells in cryptopatches, isolated lymphoid follicles, and Peyer's patches. J. Immunol. 178: 5659-5667. [Medline] [CrossRef]

26. Wood, M. B., Rios, D. and Williams, I. R. 2016. TNF- $\alpha$ augments RANKL-dependent intestinal M cell differentiation in enteroid cultures. Am. J. Physiol. Cell Physiol. 311: C498-C507. [Medline] [CrossRef]

27. Yamamoto, K., Qi, W. M., Yokoo, Y., Miyata, H., Udayanga, K. G. S., Kawano, J., Yokoyama, T., Hoshi, N. and Kitagawa, H. 2009. Histoplanimetrical study on the spatial relationship of distribution of indigenous bacteria with mucosal lymphatic follicles in alimentary tract of rat. J. Vet. Med. Sci. 71: 621-630. [Medline] [CrossRef]

28. Yuasa, H., Mantani, Y., Masuda, N., Nishida, M., Kawano, J., Yokoyama, T., Hoshi, N. and Kitagawa, H. 2017. Differential expression of Toll-like receptor-2, -4 and -9 in follicle-associated epithelium from epithelia of both follicle-associated intestinal villi and ordinary intestinal villi in rat Peyer's patches. J. Vet. Med. Sci. 78: 1797-1804. [Medline] [CrossRef]

29. Zermati, Y., Garrido, C., Amsellem, S., Fishelson, S., Bouscary, D., Valensi, F., Varet, B., Solary, E. and Hermine, O. 2001. Caspase activation is required for terminal erythroid differentiation. J. Exp. Med. 193: 247-254. [Medline] [CrossRef] 